\title{
Eine Untersuchung über die akademische Selbstwirksamkeit von Studierenden in der türkischen Hochschulbildung
}

\author{
Özdal Koyuncuoğlu (D), Konya \\ https://doi.org/10.37583/diyalog.1030828
}

\begin{abstract}
Deutsch)
Die akademische Selbstwirksamkeit ist einer der wichtigsten Faktoren, die die akademische Leistung von Studierenden in ihren Lernprozessen beeinflussen. Ziel dieser Studie ist es, die akademische Selbstwirksamkeit von Studierenden an Fakultäten, Hochschulen und Berufsfachhochschulen durch einen Vergleich nach Geschlecht, Klasse und Leistungsmerkmalen zu untersuchen. Die Studie wurde an 629 Studierenden der Universitäten Necmettin Erbakan und Kırklareli durchgeführt. Zur Erhebung der Forschungsdaten wurde eine Skala der akademischen Selbstwirksamkeit verwendet. Den Forschungsergebnissen zufolge unterscheidet sich die akademische Selbstwirksamkeit von Studierenden nach Hochschulart, Klassenstufe und schulischen Leistungsmerkmalen. Zudem wurden teilweise Unterschiede in der akademischen Selbstwirksamkeit männlicher und weiblicher Studierende beobachtet.
\end{abstract}

Schlüsselwörter: Akademische Selbstwirksamkeit, Hochschule, Berufsfachhochschule, Hochschulbildung, Türkei.

\section{Abstract (English)}

An Investigation on the Academic Self-Efficacy of Students in Turkish Higher Education

Academic self-efficacy is one of the most important factors influencing the academic performance of students in their learning processes. The aim of this study is to examine the academic self-efficacy of students at faculties, high schools and higher vocational schools by comparing them by gender, class and performance characteristics. The study was carried out on 629 students from Necmettin Erbakan and Kırklareli Universities. An academic self-efficacy scale was used to collect the research data. According to the research, the self-efficacy of students at school differed according to type of school, grade level and school performance characteristics. However, differences in the academic self-efficacy of male and female students were observed in some cases.

Keywords: Academic Self-Efficacy, Applied Sciences High School, Higher Vocational School, Higher Education, Turkey. 


\section{EXTENDED ABSTRACT}

Higher education institutions in Germany are classified as high schools (Fachhochschule) and universities. Universities of applied sciences are institutions that traditionally offer practiceoriented training in their subject areas and are usually oriented towards the applied sciences (Holuscha 2013: 273ff.). Many universities of applied sciences in Germany are now only referred to as "high schools" and are often associated with their respective subject area; for example the University of Technology and Economics. In the English language, the term "University of Applied Sciences" is used (Bachmann 2020: 1). Universities in Germany are referred to as universities enetitled to award doctorates. Universities traditionally place more value on a theoretical approach to teaching and research than universities (Holuscha 2013: 280f.). Bachelor's and master's degrees can be obtained in Germany at both high schools and universities. Contrary to what is often misunderstood, both are completely equal. Therefore, a bachelor's degree from a university is no higher than a bachelor's degree from a high school. One of the main differences between high school and universities is the style of teaching and the group size of students. While high school are generally known for a practice-oriented and school-based teaching style, compulsory internships, classes under 40 students, universities are usually characterized by a more theoretical, research-oriented and usually with over 60 students in a lecture. There is also an important difference between high school and university with regard to the admission requirements.

Faculties in Turkey are units in which scientific research and publishing activities are carried out. Different departments and programs can be located within the same faculty. Students in the faculty receive a bachelor's degree at the end of a four-year training program. The faculties are geared towards academic education. Universities, on the other hand, are defined as subordinate units to universities that concentrate on training for a specific occupation (YÖK 2019). Universities are units that train practitioners. They differ from the faculties in that they were founded for the purpose of applied education. High schools in Turkey are called "applied sciences high schools". High schools also differ more from faculties in terms of how they work: (1) The students receive practical training for certain professional fields. (2) In the eighth semester of the training process, the students go through a compulsory part-time internship. (3) Universities do not offer graduate training. Universities of applied sciences that have achieved faculty status can offer postgraduate training as a faculty of applied sciences. At universities, students receive a bachelor's degree at the end of their four-year training. There is no difference between faculty and high school in terms of diploma and title. Applied science faculties/ high schools in Turkey are similar to colleges in the US in that they are subordinate to the university as units. Universities of applied sciences in Germany, on the other hand, are independent universities. Higher vocational schools in Turkey are units that aim to train qualified workers for specific occupations and receive an associated degree at the end of the two-year training (Study in Turkey 2021; YÖK 2019).

Most of the factors influencing academic success in higher education depend on the student. At the beginning of the problems posed by the students is the fact that the students have no academic self-efficacy and no effective learning, no attitudes and habits. In fact, the student's level of learning ability in their academic process proves to be a determining factor in their school success. Indeed, one of the main factors for the success of a successful student is that the student has adequate academic knowledge and learning skills (Güngör 2010: 2). The aim of this study is to examine the academic self-efficacy of students at faculties, applied sciences high schools and higher vocational schools by comparing them by gender, class and performance characteristics. The study was carried out on 629 students from Necmettin Erbakan and 
Kırklareli Universities. An academic self-efficacy scale was used to collect the research data. According to the research, the self-efficacy of students at school differed according to type of school, grade level and school performance characteristics. However, differences in the academic self-efficacy of male and female students were observed in some cases. 


\section{Einleitung}

Im Sommersemester 2020/ 2021 waren rund 7,9 Millionen Studierende an türkischen Hochschulen immatrikuliert (ohne Fernstudium). Circa 3 Millionen dieser Studierenden sind an Hochschulen und Berufsfachhochschulen eingeschrieben. 4,9 Millionen Studierende studieren in grundständigen Studiengängen an Fakultäten (vgl. YÖK 2021).

In diesem Artikel soll die Bedeutung der Selbstwirksamkeitserwartungen von Studierenden in Bezug auf das universitäre Lernen thematisiert werden. Die Studie zielt darauf ab, die akademische Selbstwirksamkeit der Studierenden an Fakultäten, Hochschulen und Berufsfachhochschulen in der Türkei zu untersuchen, indem sie Selbstwirksamkeitserwartungen der Studierenden nach Klasse, Leistung und Geschlecht vergleicht. Zudem zeigt es auch Maßnahmen auf, die dazu beitragen können, das Erfolgserlebnis und somit die Selbstwirksamkeit von Studierenden zu fördern.

In diesem Kontext soll zunächst die Frage beantwortet werden, was der Unterschied zwischen „Universität“, „Hochschule“, „Fakultät“, „Colleges“ und „Berufsfachhochschule“ ist.

In den Vereinigten Staaten werden die beiden Begriffe Universität und Hochschule (Engl.: College) oft synonym verwendet, um sich auf Hochschuleinrichtungen zu beziehen, was bei Studierenden und Eltern gleichermaßen Verwirrung stiftet (vgl. Epps 2020: 1). Das US-amerikanische Hochschulsystem ist in Colleges und Universitäten strukturiert. Die Begriffe „Colleges“ oder „Universität“ sind jedoch kein Qualitätsindikator. Beides sind Universitäten. Colleges sind auf eine bestimmte Reihe von Programmen spezialisiert, in denen Studierende in einem bestimmten Fach studieren können. Darüber hinaus ist ein College in der Regel eine Untereinheit einer Universität. $\mathrm{Ob}$ es Universität oder Colleges heißt, hängt auch von den jeweiligen Bundesländern ab. Colleges bieten oft eine ,grundständige Ausbildung” an, die zu einem Bachelor-Abschluss führt. Studierende können dann an einer Hochschule ein „Graduate Studies“ hinzufügen und einen Master in einem Fach erwerben. Es gibt jedoch auch Hochschulen, an denen sie jeden Abschluss machen können. Universitäten bestehen in der Regel aus verschiedenen Colleges. Zusammenfassend kann man sagen, dass eine Universität aus mehreren Colleges besteht. Diese Colleges entsprechen den Fakultäten. Es besteht die Möglichkeit, an Universitäten zu promovieren. Eine Promotion an Colleges ist in der Regel nicht möglich (vgl. Holuscha, 2013: 281). Aus diesem Grund ähneln „Colleges“ in den USA den „Fachhochschulen“ in Deutschland und den Hochschulen für angewandte Wissenschaften in der Türkei (vgl. Çavuşoğlu / Günay 2010: 371). Aufgrund der unterschiedlichen Namenskonventionen in den Bundesländern kann eine Universität mancherorts auch als „College“ bezeichnet werden. Die erste Universität in den USA war die Harvard Universiät. 1636 wurden in der damaligen Kolonie Massachusetts die Oxford- und Cambridge-Modelle aus Großbritannien modelliert (vgl. Focus 2021: 1).

Hochschulen in Deutschland sind zweigeteilt. Fachhochschulen und Universitäten. Fachhochschulen sind Einrichtungen, die in ihren Fachbereichen traditionell eine praxisorientierte Ausbildung anbieten und in der Regel auf die 
angewandten Wissenschaften ausgerichtet sind (vgl. Holuscha 2013: 273ff.). Viele Fachhochschulen in Deutschland werden heute nur noch als „Hochschule“ bezeichnet und oft zusammen mit ihrem jeweiligen Fachgebiet verwendet. Zum Beispiel die Hochschule für Technik und Wirtschaft. In der englischen Sprache wird der Begriff „University of Applied Sciences“ verwendet (vgl. Bachmann 2020: 1; Holuscha 2013: 106-107). Universitäten in Deutschland werden als Hochschulen mit Promotionsrecht bezeichnet. Universitäten legen traditionell mehr Wert auf einen theoretischen Ansatz in Lehre und Forschung als Hochschulen (vgl. Holuscha 2013: 280f.). Bachelor- und Master-Abschlüsse können in Deutschland sowohl an Hochschulen als auch an Universitäten erworben werden. Im Gegensatz zu dem, was häufig missverstanden wird, sind beide völlig gleichwertig. Daher ist ein Bachelor-Abschluss einer Universität nicht höher als ein Bachelor-Abschluss einer Hochschule. Einer der Hauptunterschiede zwischen Fachhochschulen und Universität hat mit dem Unterrichtsstil und der Gruppengröße der Studenten zu tun. Während Hochschulen allgemein für einen praxisorientierten und schulischen Lehrstil, Pflichtpraktika, Klassen unter 40 Studierenden bekannt sind, zeichnen sich Universitäten in der Regel durch einen eher theoretischen, forschungsorientierten und in der Regel mit über 60 Studierenden in einer Vorlesung aus. Auch hinsichtlich der Zulassungsvoraussetzungen gibt es einen wichtigen Unterschied zwischen Hochschule und Universität. Studierende, die sich für ein Studium an einer Hochschule bewerben, müssen den Berufsausbildungsabschluss oder Berufserfahrung besitzen, während Studierende, die sich für ein Studium an einer Universität bewerben, das allgemeine Abitur (die allgemeine Hochschulreife) als Zulassungsvorraussetzung erfüllen. Da die beiden Hochschultypen unterschiedliche Lehrstile haben, unterscheidet sich auch das Angebot an Bildungsangeboten. Einige Studiengänge wie Jura oder Medizin werden auch nur an der Universität angeboten. Man kann sagen, dass die Unterschiede zwischen Hochschule und Universität im Zuge des Bologna-Reformprozesses komplexer wurden (vgl. Bachmann 2020: 1).

Das Hochschulsystem der Türkei hat gemäß dem neuen Hochschulgesetz Nr. 2547 vom 06. November 1981 einen umfassenden Umstrukturierungsprozess durchlaufen. Mit diesem Gesetz wurden alle Hochschuleinrichtungen in der Türkei an dem Hochschulrat (YÖK) untergeordnet und das System wurde zentralisiert. Akademien wurden in Universitäten umgewandelt, Bildungsinstitute wurden in Pädagogische Fakultäten umgewandelt und Berufsfachhochschulen und Konservatorien wurden an Universitäten angeschlossen (vgl. YÖK 2019). So wurden alle Hochschulen als Universitäten konzipiert. Die Hochschulbildung hat sich im ganzen Land verbreitet, die Bewerbung an den Hochschulen wurde zentralisiert und ein zentrales Hochschulprüfungs- und Einstufungssystem wurde eingeführt. Die erste gemeinnützige Stiftungsuniversität der Türkei wurde 1986 gegründet (vgl. Study in Turkey 2021).

Fakultäten in der Türkei sind universitäre Einheiten, in denen wissenschaftliche Forschungen betrieben und Publikationen veröffentlicht werden. Innerhalb der Fakultät können verschiedene Abteilungen und Programme angesiedelt sein. Studierende der Fakultät erhalten am Ende eines mindestens vierjährigen Ausbildungsprogramms einen Bachelor-Abschluss. Die Fakultäten sind auf die akademische Ausbildung ausgerichtet. 
Hochschulen hingegen werden als Universitäten untergeordnete Einheiten definiert, die sich auf die Ausbildung für einen bestimmten Beruf konzentrieren (vgl. YÖK 2019). Hochschulen sind ebenso Universitätseinheiten, die Praktiker ausbilden. Sie unterscheiden sich von den Fakultäten dadurch, dass sie zum Zweck der angewandten Ausbildung gegründet wurden. Hochschulen in der Türkei werden Hochschulen für angewandte Wissenschaften genannt. Auch in der Funktionsweise unterscheiden sich die Hochschulen stärker von den Fakultäten: (1) Die Studierenden erhalten eine praktische Ausbildung für bestimmte Berufsfelder. (2) Im Ausbildungsprozess durchlaufen die Studierenden im achten Semester ein berufsbegleitendes Pflichtpraktikum. (3) Hochschulen bieten keine Graduiertenausbildung an. Hochschulen, die den Status einer Fakultät erlangt haben, können eine postgraduale Ausbildung als Fakultät für Angewandte Wissenschaften anbieten. An Hochschulen erhalten die Studierenden am Ende der vierjährigen Ausbildung einen BachelorAbschluss. Es gibt keinen Unterschied zwischen Fakultät und Hochschule in Bezug auf Diplom und Titel. Fakultäten für angewandte Wissenschaften oder Hochschulen für angewandte Wissenschaften in der Türkei ähneln Colleges in den USA, da sie als Einheiten der Universität untergeordnet sind. Fachhochschulen in Deutschland hingegen sind eigenständige Hochschulen. Berufsfachhochschulen in der Türkei sind Einheiten, die darauf abzielen, Studierende zu qualifizierten Arbeitskräften für bestimmte Berufe auszubilden. Am Ende der zweijährigen Ausbilding erhalten die Studierenden einen assoziierten Abschluss (vgl. Study in Turkey 2021; YÖK 2019). Die meisten Berufsfachhochschulen sind an Universitäten angegliedert. Daneben gibt es Berufsfachhochschulen, die freien Stiftungen angegliedert sind, zum Beispiel Militärund Polizeischulen.

Die meisten Faktoren, die den akademischen Erfolg in der Hochschulbildung beeinflussen, hängen von den Studierenden ab. Tatsache ist, dass Studierende am Anfang ihres Studiums geringe akademische Selbstwirksamkeit und geringes effektives Lernen, nahezu keine Einstellungen und Gewohnheiten haben. Tatsächlich erweist sich das Niveau der Lernfähigkeit des Studierenden in seinem akademischen Prozess als ein bestimmender Faktor für seinen akademischen Erfolg. Einer der Hauptfaktoren für den Erfolg eines Studierenden ist es, dass der Studierende über angemessene akademische Kenntnisse und Lernfähigkeiten verfügt (vgl. Güngör 2010: 2).

\section{Akademische Selbstwirksamkeit}

Unter Selbstwirksamkeit (oder auch: Selbstwirksamkeitserwartung, Selbstregulation, Kompetenzerwartung) wird die Überzeugung einer Person verstanden, die Fähigkeit zu haben, herausfordernde Situationen aus eigener Kraft erfolgreich zu bewältigen (vgl. Bandura 1997: 3). Der wesentliche Aspekt hierbei ist die Kontrolle über das eigene Handeln und die Überzeugung, in der Lage zu sein, etwas positiv beeinflussen zu können (vgl. Bandura 1977: 3). Während Menschen mit hoher Selbstwirksamkeitserwartung schwierige Situationen und Herausforderungen in Angriff nehmen, nehmen Menschen mit niedrige Selbstwirksamkeitserwartung Aufgabe gar 
nicht erst in Angriff. Mit anderen Worten: Je kompetenter sich eine Person fühlt, desto anspruchsvoller werden ihre Ziele, und desto mehr Anstrengung und Ausdauer werden sie aufwenden, diese Ziele zu erreichen (vgl. Kriegler-Kastelic 2018: 1). Aussagen wie „Schwierigkeiten sehe ich gelassen entgegen, da ich immer auf meine eigenen Fähigkeiten vertrauen kann.“, „Wenn ein Problem auftaucht, kann ich es aus eigener Kraft meistern.“ oder „Auch bei überraschenden Ereignissen glaube ich, dass ich gut damit zurechtkommen kann." charakterisieren das Selbstwirksamkeitskonzept (vgl. Schwarzer / Jerusalem 2002: 40). Wohingegen eine Aussage wie „das schaffe ich nicht, das ist zu schwierig!“ zu einer niedriegen Selbstwirksamkeiterwartung andeutet.

Die Verbesserung und Wirksamkeit der aktuellen beruflichen Kompetenzen von Universitätsstudenten hängt weitgehend von ihren akademischen Kompetenzen in ihren Fachgebieten ab. Die Entwicklung der akademischen Selbstwirksamkeit der Studierenden hängt vom Verständnis und der Anwendung der Lehrprinzipien des Studiengangs ab. In diesem Zusammenhang hängt es eng damit zusammen, dass die Lehrinhalte von den Studierenden erworben werden. Studierende müssen ihre kognitiven Kompetenzen in der Unterrichtsumgebung in die Praxis umsetzen.

Studierende mit einem ausgeprägten Kompetenzempfinden neigen dazu, sich mit schwierigen Aufgaben selbst herauszufordern und sind hoch intrinsisch motiviert. Studierende mit einer hohen Wahrnehmung über ihre Kompetenzen fordern sich eher mit schwierigen Aufgaben heraus und sind intern motiviert. Diese Studierende bemühen sich sehr, ihrer Verantwortung nachzukommen, und führen Misserfolge auf Dinge zurück, die sie selbst kontrollieren, anstatt auf externe Faktoren zurückzuführen. Studierende mit starker Selbstwirksamkeit erholen sich schnell von Niederlagen und erreichen mit hoher Wahrscheinlichkeit ihre persönlichen Ziele. Andererseits glauben Studierende mit geringer Selbstwirksamkeit, dass sie nicht erfolgreich sein werden und ihre Aufgaben wahrscheinlich nicht erfüllen können. Daher haben Studierende mit unzureichender Selbstwirksamkeit niedrige Anforderungen, Erwartungen und Ziele, was dazu führen kann, dass enttäuschende akademische Leistungen Teil einer selbsterfüllenden Schleife werden (vgl. Margolis / McCabe 2006: 219).

Laut Maddux / Kleiman (2021: 1) bezieht sich der Begriff „Selbstwirksamkeit“ auf Überzeugungen über die Fähigkeit, die zur Erreichung eines Ziels erforderlichen Aufgaben effektiv zu erfüllen. Selbstwirksamkeit ist kein einzelnes Konstrukt. Im Gegenteil, Menschen glauben an ihre Selbstwirksamkeit in verschiedenen Bereichen wie der akademischen Selbstwirksamkeit, der Selbstwirksamkeit zur Problemlösung und der Selbstwirksamkeit zur Selbstregulation. Selbstwirksamkeit bezieht sich darauf, wie stark sie glauben, dass sie ihre Fähigkeiten einsetzen können, um ihre Ziele zu erreichen. Starke Selbstwirksamkeit ist mit positiven Ergebnissen wie besseren Noten, höherer sportlicher Leistung, glücklicheren romantischen Beziehungen und einem gesünderen Lebensstil verbunden (vgl. Erikson 2020: 1). Selbstwirksamkeit bezieht sich auf die Überzeugung darüber, was man mit den Fähigkeiten tun kann. Es handelt sich hier nicht um die Überzeugung dass man die Fähigkeit hat, sondern dass man mit der Fähigkeit Ziele erreichen kann. Selbstwirksamkeit ist auch kein Merkmal. Menschen verfügen beispielsweise über eine hohe akademische Selbstwirksamkeit, wenn sie der 
Meinung sind, dass sie über die erforderlichen Fähigkeiten verfügen, um in der Schule erfolgreich zu sein, und diese Fähigkeiten nutzen können, um sich zu übertreffen. Bandura (1997) berichtet in seiner Studie, dass die Art der Lernumgebung und Lehrmethode die Selbstwirksamkeit des Lernenden verbessern kann (vgl. Bandura 1997: 244ff.).

Der Glaube an die eigene Fähigkeit, Herausforderungen zu meistern, ist ein wichtiger Faktor für das allgemeine Wohlbefinden junger Menschen. Diese Überzeugung werden in der Literatur als Selbstwirksamkeitsüberzeugungen definiert. Die sozialkognitive Theorie nach Banduras (1977) zeigt mehrere Möglichkeiten auf, wie Selbstwirksamkeit erworben werden kann (vgl. Bandura 2012: 11ff.). (1) Selbstwirksamkeit wird dadurch aufgebaut, dass man Hindernisse überwindet und Erfolge erlebt, einen Erfolg, der von den eigenen Handlungen oder Beiträgen abhängt. (2) Es kann ebenfalls zur Selbstwirksamkeit führen, wenn man sieht, wie andere Hindernisse überwinden und Ziele erreichen. (3) Selbstwirksamkeit kann auch gefördert werden, wenn jemand einer Person sagt, dass sie kompetent ist. Die Selbstwirksamkeit hängt weniger von den tatsächlichen Fähigkeiten einer Person ab. Jedoch ist Tatsache, dass Personen mit hohen Selbstwirksamkeitsüberzeugungen mehr Beharrlichkeit zeigen, wenn sie mit Hindernissen konfrontiert sind (vgl. Bandura 1982: 123; Bandura 1986: 394). Nach Banduras (1977) sozialkognitiver Theorie leiten Selbstwirksamkeitsglauben das Verhalten sowohl direkt als auch indirekt über persönliche Ziele an das Ergebnis bestimmter Verhaltensweisen und Umwelteinflüsse (vgl. Bandura 2012: 14).

Es gibt viele Studien darüber, wie Selbstwirksamkeit für Kinder im Schulalter von Vorteil sein kann (vgl. Bandura 1977: 212ff.; Fuchs 2005: 65; Seligman 1991: 98ff.). Auch Studierende können von Selbstwirksamkeit profitieren. Studienanfänger mit hoher Selbstwirksamkeit in Bezug auf ihre Fähigkeit, im Studium erfolgreich zu sein, passen sich im ersten Jahr an die Universität besser an als solche mit geringer Selbstwirksamkeit (vgl. Chemers u.a. 2001: 55). Die Vorteile der Selbstwirksamkeit bleiben nicht nur während der Schulzeit, sondern bleiben über die Schulzeit hinaus. Schüler mit starken Überzeugungen bezüglich der Selbstwirksamkeit in Bezug auf gute Leistungen in der Schule neigen dazu, ein breiteres Spektrum an Karrieremöglichkeiten wahrzunehmen (vgl. Lent u.a. 1986: 268f.). Ebenso haben Menschen mit starken Überzeugungen ihrer Selbstwirksamkeit in Bezug auf ihre berufliche Arbeit oft eine erfolgreichere Karriere (vgl. Stajkovic / Luthans 1998: 256).

Akademische Selbstwirksamkeit bezieht sich auf die Einstellung und Überzeugung der Studierenden hinsichtlich ihrer Fähigkeit, akademische Leistung zu erbringen, sowie ihre Fähigkeit, wissenschaftliche Aufgaben zu erfüllen, und Lehrinhalte erfolgreich zu bewältigen. Es lässt sich sagen, dass die akademische Selbstwirksamkeit einer der wichtigsten Faktoren für die akademische Leistung ist (vgl. Petri 2020: 36; Schunk / Ertmer 2000: 633f.). Selbstwirksamkeitsüberzeugungen beruhen auf der akademischen Selbstwirksamkeitswahrnehmung. Selbstwirksamkeitsüberzeugungen führen dazu, dass Einzelpersonen durch Steigerung 
von Engagement, Anstrengung und Beharrlichkeit hervorragende Leistungen erbringen (vgl. Linnenbrink / Pintrich 2003: 128).

Wenn es um akademischen Erfolg geht, wird vor allem auf Selbstwirksamkeitsüberzeugungen Bezug genommen. Laut Bandura (1993: 117) ist akademische Selbstwirksamkeit, das Glauben des Studierenden an seine Fähigkeit, Handlungen im Zusammenhang mit akademischen Aktivitäten auszuführen und zu organisieren. Studierende mit hoher akademischer Selbstwirksamkeit sind kognitiv engagierter, beharrlich gegenüber akademischen Herausforderungen und bereit für Studienaktivitäten. Es wurde beobachtet, dass ein hohes akademisches Selbstwirksamkeitsniveau mit der Fähigkeit „,das Lernen zu lernen“ verbunden ist. In diesem Zusammenhang lernen Studierende, die sich als selbstwirksam wahrnehmen, auf anspruchsvollere Aufgaben zu bestehen und den Lernprozess selbst zu regulieren. Auf diese Weise wählen und verwenden Studierende unterschiedliche Lernstrategien, suchen die geeignetesten, um verschiedene Aufgabentypen zu bewältigen und bessere akademische Leistungen zu erbringen (vgl. Bandura 1997: 7; Guerreiro-Casanova / Polydoro 2011: 51ff.; Zimmerman / Cleary 2006: 52ff.). Im akademischen Kontext hat der Glaube des Studierenden an seine persönliche Kompetenz, seine eigenen Bildungsprozesse und -ergebnisse zu kontrollieren und in herausfordernden Fächern kompetent zu sein, wahrscheinlich einen großen Einfluss auf seinen Bildungswillen, seine Interessen und seine Bildungsleistung. Studierende, die von ihrer Fähigkeit zur Problemlösung oder Organisation ihrer Aufgabenerfüllung überzeugt sind, den Prozess leiten und ihre Leistung auf einem bestimmten Niveau organisieren, zeigen eine hohe Selbstwirksamkeit. Insofern wird Selbstwirksamkeit allgemein als mehrdimensionale Struktur akzeptiert, die sich zwischen mehreren Funktionsbereichen unterscheiden (vgl. Taipjutorus, Hansen/ Brown 2012: 58f.).

Studierende, die einen hohen Glauben an ihre Fähigkeit haben, akademischen Erfolg zu haben, sind im Studium tendenziell motivierter als die, die nicht an ihre Fähigkeit glauben (vgl. Schunk 1991: 207ff.). Wenn Studierende mit hoher Selbstwirksamkeit ihre Ziele erreichen, neigen sie dazu sich das Ziel zu setzen, anspruchsvollere Aufgaben zu erfüllen (vgl. Schunk 1990: 80). All dies kann zu einem akademischen Erfolg in Bezug auf höhere Noten und die Wahl anspruchvoller Kurse führen (vgl. Multon / Brown / Lent 1991: 30). Zum Beispiel können Studierende mit hoher akademischer Selbstwirksamkeit mehr lernen, weil sie glauben, dass sie ihre Fähigkeiten effektiver einsetzen können. Sie bekommen bei ihren nächsten Prüfungen hohe Punktzahlen, weil sie viel gelernt haben. Die Selbstwirksamkeit von Lehrkräften kann sich auch auf die Leistung der Studierenden im Studium auswirken. Selbstwirksame Lehrkräfte können zu besseren akademischen Leistungen führen, indem sie die Studierenden ermutigen, eine aktivere Rolle beim Lernen zu übernehmen (vgl. Hoover-Dempsey / Bassler / Brissie 1987: 430).

Laut Bandura (1986: 25) bezieht sich Selbstwirksamkeit auf die Wahrnehmung, die die Person über ihre eigene Fähigkeit hat, die vorgeschlagene Aktivität zu erreichen. In diesem Wahrnehmungsprozess interpretieren die Studierenden die Ergebnisse ihrer Aktivitäten und akademischen Aufgaben. In diesem Zusammenhang können sich die 
Studierenden in ihren Ansichten über Lernfähigkeiten oder in Bezug auf die „effektive“ Anpassung an den Lernkontext unterscheiden (vgl. Schunk 1991: 211; Piergiovanni / Depaula 2018: 18). In diesem Sinne scheinen beide Konstrukte einen gemeinsamen Effekt auf die Entwicklung allgemeiner und akademischer Selbstwirksamkeit, akademischer Leistungen und adaptiver akademischer Ziele zu haben (vgl. Pajares 1996: 543f.; Valentine u.a. 2004: 113; Wolf u.a. 2018: 42). Obwohl akademische Selbstwirksamkeit ein wichtiges Merkmal im Bildungsbereich ist, kann die allgemeine Selbstwirksamkeit jedoch eine globalere Perspektive auf die Selbstwahrnehmung der Studierenden in den vielen stressigen Kontexten des täglichen Lebens bieten (vgl. Sanjuán u.a. 2000: 509). Mit anderen Worten, obwohl Selbstwirksamkeit als spezifischer Einflussbereich angesehen wird, kann sie als eine höhere oder niedrigere Selbstwirksamkeit interpretiert werden, um Vertrauen oder allgemeines Urteilsvermögen zu beschreiben, wenn Studierende neuen oder stressigen Situationen begegnen (vgl. Luszczynska u.a. 2005: 81; Bueno-Pacheco u.a. 2017: 6).

Ormrod, Anderman und Anderman (2017: 386f.) sind der Meinung, dass die akademische Selbstwirksamkeit die Gedanken, Muster und die emotionale Erregung beeinflusst, die zu vorhergesagten akademischen Handlungen führen. In dieser Hinsicht haben die Wahrnehmungen der akademischen Selbstwirksamkeit direkte und indirekte Auswirkungen auf das menschliche Verhalten in einer Weise, die sich auf das Engagement der Studierenden für akademische Ziele, die akademischen Entscheidungen der Studierenden und ihre Gefühle gegenüber Schwierigkeiten und deren Förderer in Bezug auf akademische Ziele auswirkt (vgl. Zimmerman / Kitsantas 2005: 513f.; Blanco u.a. 2011, zit. nach Musa 2020: 136)

Akademische Selbstwirksamkeit ist ein Schlüsselmerkmal für den Studienerfolg von Hochschulstudenten (vgl. Hill 2002: 121f.). Zimmerman (2000: 83) betont, dass akademische Selbstwirksamkeit ein multidimensionales Merkmal ist und nicht als einzelne Disposition erscheint. Demzufolge wird die allgemeine Selbstwirksamkeit oft in zwei Stufen eingeteilt. (1) Die akademische Selbstwirksamkeit und (2) die kognitive Fähigkeiten. Akademische Selbstwirksamkeit fokussiert sich auf die wahrgenommene akademische Fähigkeit des Studierenden, anspruchsvolle akademischen Aufgaben zu erfüllen. Es wird von Studierenden mit hoher akademischer Selbstwirksamkeit erwartet, dass sie hohe akademische Leistung erbringen (vgl. Mehmood u.a. 2019: 33; Murphy / Alexander 2000: 41). Nach Blanco u.a. (2011) bedeutet akademische Selbstwirksamkeit, überzeugt zu sein, die erforderlichen Kompetezen entwickeln zu müssen, um akademische Ziele erreichen zu können. Blanco u.a. (2011) schlagen vor, dass das akademische Selbstwirksamkeitskonzept drei Dimensionen beinhaltet (Musa 2020: 137): (1) Aufmerksamkeit, (2) Kommunikation und (3) Exzellenz. Aufmerksamkeit ist die Konzentration der Studierenden auf akademische Aufgaben. Dies hilft den Studierenden, ihre Schwierigkeiten wahrzunehmen und dann auf Schwachstellen zu achten, um erfolgreich zu sein (vgl. Fuenmayor / Villasmil 2008: 193). Kommunikation besteht aus Elementen wie psychoedukativen Fähigkeiten, Psycholinguistik, Metakognition und soziokulturellen Fähigkeiten, die den Lernprozess erleichtern können (vgl. Valdivieso / Carbonero / Martin 2013: 66). Exzellenz erfordert 
jedoch Fähigkeiten und Kompetenzen, die es ermöglichen, Standards und Normen einzuhalten, um hohe akademische Leistungen zu erzielen. Exzellenz ist ein Produkt aus erreichbaren Zielen und der Entwicklung geeigneter Strategien und Pläne, die Studierende zu den gewünschten Zielen führen können (vgl. Herrera 2013: 89f.). Akademische Selbstwirksamkeit kann auf akademischen Erfahrungen, persönlichen Herausforderungen, zwischenmenschlichen Beziehungen der Studierenden beruhen. Ebenso kann akademische Selbstwirksamekeit von den Studien- oder Studierendenbewertungsmethoden, Studienstrategien für Studierende und institutioneller Bewertung abhängen. Kognitive Fähigkeiten und akademische Selbstwirksamkeit wurden als Prädiktoren für die akademischen Leistungen der Studierenden bestimmt. Das akademische Selbstwirksamkeitskonzept betont, dass das Vertrauen des Einzelnen in seine Fähigkeit, bestimmte Ziele zu erreichen, ihn dazu antreibt, auch bei anspruchsvollen Aufgaben maximale Leistung und Erfolg anzustreben (vgl. Mehmood u.a. 2019: 43).

Es werden zwei allgemeine Kategorien der akademischen Erwartungshaltung vorgeschlagen. Akademische Ergebniserwartungen sind die Überzeugung eines Studierenden, dass bestimmte Verhaltensweisen zu bestimmten Ergebnissen führen (z. B. „Meine Noten verbessern sich, wenn ich Hausaufgaben mache”). Akademische Leistungserwartungen sind Überzeugungen über die Fähigkeit eines Studierenden, die Verhaltensweisen auszuführen, die zum Erreichen eines bestimmten Ergebnisses erforderlich sind (z. B. „Ich habe genug Motivation, um für diesen Test hart zu lernen”). Den Unterschied zwischen diesen beiden Formen der Erwartungshaltung zu verstehen bedeutet, dass Personen vielleicht glauben, dass ein bestimmtes Verhalten zu einem bestimmten Ergebnis führt (Ergebniserwartung), aber nicht, dass sie dieses Verhalten ausführen können (Aktivitätserwartung) (vgl. Eccles / Wigfield 2002: 111). Linnenbrink und Pintrich (2003: 124ff.) haben mit ihrer Untersuchung nachgewiesen, dass akademische Selbstwirksamkeit signifikant mit dem Lernen, analytischem Denken, akademischem Engagement, Strategieeinsatz, kognitivem Engagement, Beharrlichkeit, Anfälligkeit für negative Emotionen und Erfolg verbunden ist.

Mit der Darlegung der Bedeutung der Selbstwirksamkeitserwartungen konzentriert sich dieser Artikel auf die Beantwortung folgender Fragen:

- Unterscheidet sich die akademische Selbstwirksamkeit von Studierenden an Fakultät/ Hochschule und Berufsfachhochschulen?

- Unterscheidet sich die akademische Selbstwirksamkeit der Teilnehmenden je nach Geschlechtsvariable?

- Unterscheidet sich die akademische Selbstwirksamkeit der Teilnehmenden je nach Jahrgangsstufe?

- Unterscheidet sich die akademische Selbstwirksamkeit der Teilnehmenden nach ihrem Studienerfolg? 


\section{Methodische Vorgehensweise}

In dieser Studie soll untersucht werden, inwieweit die akademische Selbstwirksamkeit der Studierende im Rahmen von Fakultät/ Hochschule und Berufsfachhochschule sowie die demografischen Variablen erklären lässt. Dazu wurden die Unterschiede zwischen den Forschungsvariablen auf die abhängige Variable mit einem vergleichenden Ansatz untersucht. In diesem Zusammenhang wurde in der Studie das Modell der KausalVergleichsforschung verwendet. Diese Methode basiert auf einem Vergleich. Bei dieser Methode wird zwischen zwei oder sogar mehreren Variablen verglichen, um ihre Ursache-Wirkungs-Beziehung herauszufinden (Cohen / Manion 1994: 501ff.).

Die Forschungsdaten wurden online mithilfe eines Google-Fragebogens erhoben. Während des Datenerhebungsprozesses wurden die Teilnehmer gebeten, das am Ende des akademischen Jahres 2020-2021 über das Google-Formular erstellte und elektronisch übermittelte Formular auszufüllen. Innerhalb des erhaltenen Datensatzes wurden 629 geeignete Daten für die statistische Analyse verwendet.

Die Gesamtmenge (Population) der Studie sind die Studenten an türkischen Universitäten. Die in die Stichprobe einbezogenen Studierenden der Fakultät/ Hochschule für Angewandte Wissenschaften und Berufsfachhochschulen in den Provinzen Konya und Kurklareli. Die Auswahl dieser Regionen dient der einfacheren Durchführung der Umfrage. Die Universität Kırklareli wurde 2007 gegründet. Die Necmettin Erbakan Universität wurde 2010 gegründet. Der Zweck der Auswahl dieser Universitäten besteht darin, den Unterschied zwischen ihnen und der Ansicht zu zeigen, dass sie das Land im Allgemeinen repräsentieren können, indem Beispiele von zentralen, neuen und Provinzuniversitäten herangezogen werden. Die Forschungsgruppe besteht aus insgesamt 629 Universitätsstudenten verschiedener Klassen, 385 weibliche und 244 männliche Studierende wurden als Stichprobe herangezogen.

\section{Datenerfassungstool}

In der Datenerhebungsphase der Studie wurde die von Kandemir und Özbay (2010: 201ff.) entwickelte „Academic Self-Efficacy Scale“ verwendet. Bei der Analyse der Forschungsdaten wurde die Software SPSS 22.00 verwendet. Cronbach Alpha, Independent Samples $t$ Test und univariate Varianzanalyse (one way analysis of variance ANOVA) Datenanalysetechniken wurden herangezogen. Die Ergebnisse der empirischen Erhebung wurden dann in den Kontext der bestehenden Literatur gesetzt und diskutiert. Anschließend wurden die Forschungsfragen explizit beantwortet und auf Limitationen und Anregungen an Forscher und Praktiker für zukünftige Arbeiten eingegangen.

\section{Ergebnisse der empirischen Erhebung}

In diesem Abschnitt werden die Ergebnisse der empirischen Untersuchung dargestellt. Die empirische Forschung unterscheidet sich von anderen wissenschaftlichen Methoden 
dadurch, dass empirische Sätze an der Wirklichkeit überprüft werden können (vgl. Hug / Poscheschnik 2015: 22ff.). In der empirischen Untersuchung wurde das Modell der Kausal-Vergleichsforschung verwendet (Cohen / Manion 1994: 501ff.). Im Anschluss werden die Forschungsfragen beantwortet und diskutiert.

\begin{tabular}{|c|c|c|c|c|c|}
\hline & $\mathrm{N}$ & Minimum & Maximum & Mean & $\begin{array}{l}\text { Std. } \\
\text { Deviation }\end{array}$ \\
\hline Bewältigung & 629 & 1,00 & 5,00 & 3,80 & 0,78 \\
\hline Anstrengung & 629 & 1,00 & 5,00 & 3,95 & 0,79 \\
\hline Planung & 629 & 1,00 & 5,00 & 3,71 & 0,82 \\
\hline $\begin{array}{l}\text { Akademische } \\
\text { Selbstwirksamkeit }\end{array}$ & 629 & 1,00 & 5,00 & 3,81 & 0,75 \\
\hline
\end{tabular}

Tab. 1: Deskriptive Statistik zu akademischen Selbstwirksamkeitswerten von Universitätsstudenten

Tabelle 1 zeigt, dass die akademischen Selbstwirksamkeitsbewertungen im Bereich von 1,00 bis 5,00 liegen. Die akademische Selbstwirksamkeit der Studierenden ist nach den erzielten Durchschnittswerten in allen Subdimensionen insgesamt hoch.

\begin{tabular}{|c|c|c|c|c|c|c|}
\hline & Akademik Birim & $\mathrm{N}$ & Mean & $\begin{array}{l}\text { Std. } \\
\text { Deviation }\end{array}$ & $\mathrm{t}$ & $\mathrm{P}$ \\
\hline \multirow[t]{2}{*}{ Bewältigung } & Fakultät/ Hochschule & 344 & 3,93 & 0,71 & 4,68 & 0,00 \\
\hline & Berufsfachhochschule & 285 & 3,64 & 0,82 & & \\
\hline \multirow[t]{2}{*}{ Anstrengung } & Fakultät/ Hochschule & 344 & 4,10 & 0,69 & 5,28 & 0,00 \\
\hline & Berufsfachhochschule & 285 & 3,77 & 0,87 & & \\
\hline \multirow[t]{2}{*}{ Planung } & Fakultät/ Hochschule & 344 & 3,84 & 0,78 & 4,46 & 0,00 \\
\hline & Berufsfachhochschule & 285 & 3,55 & 0,84 & & \\
\hline \multirow{2}{*}{$\begin{array}{l}\text { Akademische } \\
\text { Selbstwirksamkeit }\end{array}$} & Fakultät/ Hochschule & 344 & 3,94 & 0,68 & 5,00 & 0,00 \\
\hline & Berufsfachhochschule & 285 & 3,65 & 0,80 & & \\
\hline
\end{tabular}

Tab. 2: Vergleich der akademischen Selbstwirksamkeit von Studierenden nach akademischen Einheiten

Tabelle 2 zeigt die Ergebnisse der Analyse der akademischen Selbstwirksamkeitsscores von Studierenden an Fakultäten/ Hochschulen und Berufsfachhochschulen. Es gibt signifikante Unterschiede in den akademischen Selbstwirksamkeitswerten der Studierenden nach der Einheit, die sie studieren $(p>0,05)$. Die wissenschaftliche Selbstwirksamkeit von Universitätsstudenten an Fakultäten/ Hochschulen war signifikant höher. 


\begin{tabular}{lllllll}
\hline & Geschlecht $\mathrm{N}$ & Mean & $\begin{array}{l}\text { Std. } \\
\text { Deviation }\end{array}$ & $\mathrm{p}$ \\
& weiblich & 385 & 3,68 & 0,78 & $-2,01$ & 0,046 \\
Bewältigung & männlich & 244 & 3,93 & 0,75 & & \\
Anstrengung & weiblich & 385 & 3,94 & 0,80 & $-0,24$ & 0,81 \\
& männlich & 244 & 3,96 & 0,77 & & \\
Planung & weiblich & 385 & 3,71 & 0,83 & $-0,34$ & 0,74 \\
& männlich & 244 & 3,73 & 0,80 & & \\
\hline Akademische & weiblich & 385 & 3,80 & 0,76 & $-0,63$ & 0,53 \\
Selbstwirksamkeit & männlich & 244 & 3,84 & 0,73 & & \\
& & & & & & \\
& & &
\end{tabular}

Tab. 3: Vergleich der akademischen Selbstwirksamkeit von Studierenden nach Geschlecht

Tabelle 3 zeigt, dass die Werte für Anstrengung, Planung und gesamte akademische Selbstwirksamkeit der Teilnehmer keinen signifikanten Unterschied in Bezug auf die Geschlechtsvariable $(p>0,05)$ aufweisen. In der Bewältigungsdimension wurde jedoch ein signifikanter Geschlechtsunterschied festgestellt. Es ist ersichtlich, dass männliche Universitätsstudenten eine signifikant höhere akademische Selbstwirksamkeit in der Bewältigungsdimension aufweisen.

\begin{tabular}{llllllc}
\hline & Jahrgangsstufe & $\mathrm{N}$ & Mean & $\begin{array}{l}\text { Std. } \\
\text { Deviation }\end{array}$ & $\mathrm{F}$ & $\mathrm{p}$ \\
\hline Bewältigung & 1,0 & 199 & 3,68 & 0,78 & 8,098 & 0,000 \\
& 2,0 & 242 & 3,73 & 0,77 & & \\
& 3,0 & 102 & 3,92 & 0,69 & & \\
Anstrengung & 4,0 & 86 & 4,11 & 0,78 & & \\
& 1,0 & 199 & 3,83 & 0,84 & 7,474 & 0,000 \\
& 2,0 & 242 & 3,87 & 0,78 & & \\
Planung & 3,0 & 102 & 4,13 & 0,68 & & \\
& 4,0 & 86 & 4,22 & 0,73 & & \\
& 1,0 & 199 & 3,58 & 0,79 & 8,519 & 0,000 \\
& 2,0 & 242 & 3,65 & 0,84 & & \\
& 3,0 & 102 & 3,82 & 0,76 & & \\
& 4,0 & 86 & 4,07 & 0,79 & &
\end{tabular}




$\begin{array}{lllllll}\text { Akademische } & 1,0 & 199 & 3,69 & 0,76 & 8,816 & 0,000 \\ \text { Selbstwirksamkeit } & 2,0 & 242 & 3,74 & 0,75 & & \\ & 3,0 & 102 & 3,94 & 0,66 & & \\ & 4,0 & 86 & 4,13 & 0,75 & \end{array}$

Tab. 4: Vergleich der akademischen Selbstwirksamkeit von Universitätsstudenten nach Jahrgangsstufen

Tabelle 4 veranschaulicht, dass es einen signifikanten Unterschied $(p<0,05)$ in den Werten für Bewältigung, Anstrengung, Planung und gesamte akademische Selbstwirksamkeit gemäß der Klassenstufenvariable gibt. Der Tukey-Test, ist ein statistischer Test, mit dem zwei unabhängige Stichproben auf Unterschiede miteinander verglichen werden können (Tukey 1959: 31). Laut der Tukey-Testanalyse sind die Mittelwerte der akademischen Selbstwirksamkeit der Studierenden der 4. und 3. Klasse signifikant höher als die Mittelwerte der Studierenden der 1. und 2. Klasse.

\begin{tabular}{lllllll}
\hline & Leistung & $\mathrm{N}$ & Mean & $\begin{array}{l}\text { Std. } \\
\text { Deviation }\end{array}$ & $\mathrm{F}$ & $\mathrm{p}$ \\
\hline Bewältigung & Hoch & 139 & 4,19 & 0,70 & 35,78 & 0,00 \\
& Mittel & 472 & 3,71 & 0,73 & & \\
& Niedrig & 13 & 2,75 & 1,17 & & \\
Anstrengung & Hoch & 139 & 4,29 & 0,73 & 30,74 & 0,00 \\
& Mittel & 452 & 3,87 & 0,74 & & \\
Planung & Niedrig & 33 & 2,85 & 1,34 & & \\
& Hoch & 139 & 4,10 & 0,73 & 29,22 & 0,00 \\
& Mittel & 472 & 3,62 & 0,79 & & \\
& Niedrig & 13 & 2,79 & 1,19 & & \\
Akademische & Hoch & 139 & 4,19 & 0,68 & 36,80 & 0,00 \\
Selbstwirksamkeit & Mittel & 452 & 3,73 & 0,71 & & \\
& Niedrig & 33 & 2,78 & 1,17 & & \\
\hline
\end{tabular}

Tab. 5: Vergleich der akademischen Selbstwirksamkeit von Universitätsstudenten nach Studienerfolg

Betrachtet man Tabelle 5, so zeigt sich ein signifikanter Unterschied in allen Dimensionen und Summen der akademischen Selbstwirksamkeitsskala nach der schulischen Erfolgsvariable $(p<0,05)$. Laut der Tukey-Testanalyse wurde festgestellt, dass die akademische Selbstwirksamkeit von Studierenden mit hohem Erfolgsniveau signifikant höher war als die durchschnittlichen Werte von Studierenden mit mäßigen und geringen Erfolgen. 


\section{Diskussion, Fazit und Empfehlungen für zukünftige Arbeiten}

In dieser Studie, in der die akademische Selbstwirksamkeitswahrnehmung von Studierenden in Bezug auf einige Variablen untersucht wurde, fielen die Bewertungen der Teilnehmer in Bezug auf die relevanten Variablen moderat aus. Ein weiteres Ergebnis dieser Studie ist, dass die Wahrnehmung der akademischen Selbstwirksamkeit von Universitätsstudenten je nach Geschlecht unterschiedlich ist. Nach den Ergebnissen der Analyse erreichten männliche Studierende höhere Durchschnittswerte in der Erfolgsdimension der akademischen Selbstwirksamkeit als ihre weiblichen Mitstudierenden. Die Ergebnisse der empirischen Erhebung zeigen, dass es im Zusammenhang der akademischen Selbstwirksamkeit der Studierenden einen signifikanten Geschlechtsunterschied gibt, der sich auf ihre akademischen Leistungen auswirken kann. Studien zufolge weisen Studierende mit hoher akademischer Selbstwirksamkeit tendenziell höheren akademischen Erfolg auf als ihre Mitstudierenden mit geringer akademischer Selbstwirksamkeit (vgl. Yokoyama 2019: 2; Ahmad / Safaria 2013: 25ff; Aurah 2017: 149ff. ; Oyuga / Raburu / Aloka 2019: 43ff.). In dieser Studie wurde festgestellt, dass auch die akademischen Leistungen von männlichen Studierenden mit höherer akademischer Selbstwirksamkeit höher waren. Dies bestätigt Erkenntnisse aus früheren Studien, die besagen, dass die Leistungen des Studierenden im Unterricht umso besser ist, je höher die akademische Selbstwirksamkeit bei Studierenden empfunden wird. Die vorherige Analogie spiegelt nur die Ergebnisse dieser Studie und Stichprobe wider, da die diskutierten Ergebnisse deskriptive Statistiken dieser Studie sind.

Ein weiteres Ergebnis der Studie bezieht sich auf die Unterschiede in der akademischen Selbstwirksamkeit zwischen Fakultäts- /Hochschul- und Berufsfachhochschulstudierenden. Nach den Ergebnissen der Studie erreichten Studierende der Fakultät/ Hochschule in allen Dimensionen der akademischen Selbstwirksamkeitsskala signifikant höhere Durchschnitte. Darüber hinaus unterscheidet sich die akademische Selbstwirksamkeit der Studierenden je nach Jahrgangsstufe deutlich. Generell zeigten Studierende der 3. und 4. Klasse eine hohe akademische Selbstwirksamkeit in den Gesamtwerten und Subdimensionen der Skala. Dabei ist zu beobachten, dass die schulische Selbstwirksamkeit mit zunehmender Klassenstufe deutlich zunimmt. Nach Schunk und Zimmerman (2006: 356ff.), Schunk und Pajares (2009: 35ff.) beeinflussen die Selbstwirksamkeit bei der Entwicklung kognitiver, affektiver und psychomotorischer Kompetenzen von Individuen und der Entwicklung domänenspezifischer Kompetenzen. Daher entwickelt sich im Laufe des akademischen Lehrnprozesses ein Bewusstsein für die grundlegenden Fähigkeiten und Kompetenzen, die die akademische Selbstwirksamkeit unterstützen.

Als letztes Ergebnis dieser Studie geht es um den Zusammenhang zwischen den Studiumleistungen der Studierenden in ihren Fachbereichen und ihrer akademischen Selbstwirksamkeit. Im Allgemeinen wurde beobachtet, dass Studierende mit sehr hohem Leistungsniveau eine höhere akademische Selbstwirksamkeitswahrnehmung haben als ihre schwachen und mäßig erfolgreichen Freunde. Mit steigenden Leistungen der Studierenden steigt ihre akademische Selbstwirksamkeit. Die Befunde dieser Studie 
ergeben neue Erkenntnisse im Bereich der akademischen Selbstwirksamkeit und der akademischen Leistungsfähigkeit von Studenten im tertiären Bereich. Die Ergebnisse dieser Studie zeigten, dass die akademische Selbstwirksamkeit der Studierenden ein entscheidender Faktor bei der Förderung der Konzentrationsfähigkeit und des Strebens nach höherer akademischer Exzellenz ist (vgl. Njega u.a. 2019: 48; Fong / Yuen 2016: $165)$.

Laut Namubiru (2019: 2ff.) steigen die akademischen Leistungen und Kompetenzen der Studierenden in dem Maße, in dem die Lehrkräfte die akademische Selbstwirksamkeit der Studierenden entwickeln. Die Studie zeigt eine Lücke auf, dass es Unterschiede zwischen Studierenden an Universitäten in Bezug auf die akademische Selbstwirksamkeit geben kann, die sich auf ihre akademischen Leistungen auswirken könnten. Demzufolge kann vorgeschlagen werden, Lehr-Lern-Methode anzuwenden, um die Studierenden bei der Entwicklung ihrer akademischen Selbstwirksamkeit zu unterstützen. Der Prozess der Entwicklung der Selbstwirksamkeit der Studierenden sollte eine aktive Beteiligung zwischen Studierenden und Lehrkräften umfassen, die unter anderem Anleitungs- und Beratungsaktivitäten umfassen können (vgl. Njega u.a. 2019: 58; Nkechi u.a. 2016: 41). Der Prozess der Gestaltung der Selbstwirksamkeit der Studierenden sollte auch die Entwicklung von Verhalten und Persönlichkeit einschließen, um sicherzustellen, dass die akademischen Fähigkeiten und Potenziale der Studierenden vollständig auf das angestrebte akademische Ziel ausgerichtet sind (vgl. Nkechi u.a. 2016: 42ff.). Die Gestaltung und Entwicklung der akademischen Selbstwirksamkeit der Studierenden sollte sowohl für Männer als auch für Frauen und in allen Studiengängen Priorität haben. Diese Studie deckt signifikante geschlechtsspezifische Unterschiede in der akademischen Selbstwirksamkeit von Studierenden an Universitäten auf. Fakultätsmitglieder haben wichtige Aufgaben und Initiativen bei der Entwicklung der akademischen Selbstwirksamkeit der Studierenden an Universitäten. In diesem Zusammenhang können Lehrkräfte verschiedene Methoden anwenden, um den Studierenden zu helfen, ihre Ziele zu erreichen. Unter den Methoden, die verwendet werden können sind u.A. wie folgt: Unterstützung von Freunden anderer Studierender, bei denen eine hohe akademische Selbstwirksamkeit festgestellt wurde, durch Teamarbeit, Kontakt der Studierenden mit Menschen oder Vorbildern mit hoher akademischer Selbstwirksamkeit, Mentoring, Anleitung und Beratung, Entwicklung umfassender Programme zum Aufbau von Kapazitäten. All dies wird dazu beitragen, die akademische Selbstwirksamkeit der Studierenden zu gestalten und zu verändern.

An Universitäten sollen die akademischen Qualifikationen der Studierenden ermittelt und Aktivitäten organisiert werden, die die Kandidaten auf ein ausreichendes Niveau bringen können. Hochschulstudierende sollen sich dort bewerben, wo sie ihre lernwirksamen und akademischen Kompetenzen in ihren Fachgebieten während ihres Studiums einsetzen können, insbesondere um die fachbezogenen wissenschaftlichen Kenntnisse umzusetzen. Hierzu können in den Hochschulprogrammen notwendige Vorkehrungen getroffen werden. Studierende sollen durch Konferenzen zu effektivem Lernen und schulischer Selbstwirksamkeitsentwicklung in Fakultäten/ Hochschulen und 
Berufsfachhochschulen aufgeklärt werden. Berater können zur Entwicklung der akademischen Selbstwirksamkeit der Studierenden beitragen, indem sie den Studierenden eine effektive akademische Beratung bieten. Darüber hinaus können standardisierte Fragebögen eingesetzt werden, die versuchen, die akademischen Kenntnisse und Fähigkeiten der Studierenden zu Beginn und am Ende der Hochschulausbildung valide und verlässlich zu bewerten.

Aus den Ergebnissen dieser mit quantitativen Methoden durchgeführten Forschung lassen sich einige Anregungen für die Ausbildung und Forschung von Studierenden an Hochschulen geben. Zuallererst sollten an Universitäten Programme angeboten werden, die den Studierenden professionelles Wissen, Techniken zur Anwendung des erlernten Wissens, nicht nur professionelles Verhalten, sondern auch akademische Kompetenzen und Fähigkeiten vermitteln. Es wird empfohlen, Studierende mit hoher akademischer Selbstwirksamkeit und Lernkompetenz auf der Grundlage der Ausrichtung auf eine akademische Laufbahn mit den Kapazitäten ihrer Fakultäten an Universitäten zu fördern. Eine Vielzahl von Studien existiert, die den Einfluss von Variablen im Zusammenhang mit der Selbstwirksamkeit von Studierenden untersucht haben. Es gibt jedoch eine gerine Anzahl an Studien, die die Entwicklung der akademischen Selbstwirksamkeit von Universitätsstudenten und deren Auswirkungen auf den Lehrnprozess widerspiegeln. Aus diesem Grund können experimentelle und Längsschnittstudien durchgeführt werden, um die akademische Selbstwirksamkeit von Universitätsstudenten während ihrer Bachelor- und Masterausbildung zu entwickeln und zu überprüfen.

\section{Literaturverzeichnis}

Ahmad, Alay / Safaria, Triantoro (2013): Effects of self-efficacy on students' academic performance. In: Journal of Educational, Health and Community Psychology 2(1), 22-29.

Aurah, Catherine (2017): Investigating the Relationship between Science Self-efficacy Beliefs, Gender, and Academic Achievement, among High School Students in Kenya. In: Journal of Education and Practice, 8(8), 146-153.

Bachmann, Hannah (2020): Die Unterschiede zwischen Hochschule und Universität. https://www.scribbr.de/studium/hochschule-universitaet/ (Letzter Zugriff: 15.07.2021).

Bandura, Albert (1977): Self-efficacy: Toward a unifying theory of behavioral change. Psychological Review 84(2), 191-215. doi: 10.1037/0033-295X.84.2.191.

Bandura, Albert (1982): Self-efficacy mechanism in human agency. American Psychologist 37(2), 122147.

Bandura, Albert (1986): Social foundations of thought and action: A social cognitive theory. Englewood Cliffs, NJ: Prentice Hall.

Bandura, Albert (1993): Perceived self-efficacy in cognitive development and functioning. In: Educational Psychologist, 28(2), 117-148.

Bandura, Albert (1997): Self-efficacy, the exercise of control. New York: Freeman and Company.

Bandura, Albert (1998): Health promotion from the perspective of social cognitive theory. Psychology \& Health 13(4), 623-649. doi: 10.1080/08870449808407422. 
Bandura, Albert (2006). Guide for Constructing Self-Efficacy Scales. In: Pajares, Frank/ Urdan, Tim (Hg.): Self-Efficacy Beliefs of Adolescents. Greenwich, CT: Information Age Publishing, 5, 307337.

Bandura, Albert (2012): On the functional properties of perceived self-efficacy revisited. Journal of Management 38(1), 9-44. doi: 10.1177/0149206311410606.

Blanco, Humberto / Martinez, M. A. / Ornelas, Martha / Flores, Francisco J. / Peinado, Jesus E. (2011): Validación de las escalas de autoeficaciaenconductasacadémicas y cuidado de la salud. México: DobleHélice.

Bueno-Pacheco, Alexandra / Lima-Castro, Sandra / Peña-Contreras, Eva / Cedillo-Quizhpe, Cristina / Aguilar-Sizer, Monica (2017): Spanish adaptation of the general self-efficacy scale for use in Ecuadorian context. RIDEP, 3, 5-15. 10.21865/RIDEP48.3.01.

Çavuşoğlu, Abdullah / Günay, Durmuş (2010): The reforming of vocational teacher training colleges in Turkey. In Lytras, Miltiadis D. u.a. (Hg.): Technology Enhanced Learning. Quality of Teaching and Educational Reform. TECH-EDUCATION 2010. Communications in Computer and Information Science, 73. Berlin und Heidelberg: Springer,. 364-373. https://doi.org/10.1007/9783-642-13166-0_52.

Chemers, Martin M. / Hu, Li-tze / Garcia, Ben F. (2001): Academic self-efficacy and first year college student performance and adjustment. In: Journal of Educational Psychology, 93(1), 55-64. doi:10.1037/0022-0663.93.1.55.

Cohen, Louis / Manion, Lawrence (1994). Research methods in education. London/ New York: Routledge.

Eccles, Jacquelynne S. / Wigfield, Allan (2002): Motivational beliefs, values, and goals. In: Annu. Rev. Psychol., 53, 109-32.

Epps, Tyler (2020): College vs. University: What's the Difference? https://www.bestcolleges.com/blog/difference-between-college-and-university/ (Letzter Zugriff 15.07.2021).

Erikson, Erik (2020): Psychosocial Development in Middle Childhood - Self Concept and Self Efficacy. https://socialsci.libretexts.org/Courses/Foothill_College/

Psych_40\%3A_Lifespan_Development_(Pilati)/06\%3A_Middle_Childhood/6.05\%3A_Psychosoci al_Development_in_Middle_Childhood_-_Self_Concept_and_Self_Efficacy (Letzter Zugriff: 15.07.2021).

Focus (2021): Was ist der Unterschied zwischen College und University? Einfach erklärt. https://praxistipps.focus.de/was-ist-der-unterschied-zwischen-college-und-university-einfacherklaert_96722 (Letzter Zugriff: 01.07.2021).

Fong, Ricci W. / Yuen, Man T. (2016): The Role of Self-Efficacy and Connectedness in the Academic Success of Chinese Learners. King, Ronnel B./ Bernardo, Allan B. (Hg.): The Psychology of Asian Learners. Singapore: Springer, 157-167. doi: 10.1007/978-981-287-576-1_10.

Fuchs, Carina (2005): Selbstwirksam lernen im schulischen Kontext. Kennzeichen - Bedingungen Umsetzungsbeispiele. Bad Heilbrunn : Klinkhardt.

Fuenmayor, Gloria / Villasmil, Yeriling (2008): La percepción, la atención y la memoria como procesos cognitivos utilizados para la comprensión textual. In: Revista de Artes y Humanidades UNICA 9(22), 187-202.

Guerreiro-Casanova, Daniela / Polydoro, Soely A. J. (2011): Self-efficacy in higher education: Perceptions during the first year of graduation. In: Psicologia: Ciência e Profissão 31(1), 50-65.

Güngör, Gözde (2010): İlköğretim düzeyindeki öğrencilerin benlik kavramlarının ve çalışma alışkanlıklarının değerlendirilmesi. (Unveröffentlichte Masterarbeit). Gazi Üniversitesi, Ankara. 
Hattie, John (2015): Lernen sichtbar machen, 3., Aufl. Baltmannsweiler: Schneider-Verlag Hohengehren.

Herrera, Lucía (2013): Conceptualizacion academica de la excelenciaen el ambitouniversitario. In: Omnia, 19(1), 86-98.

Hill, Winfred F. (2002): Learning: A Survey of Psychological Interpretations (7th edn.). Boston: Allyn and Bacon.

Holuscha, Elisabeth (2013): Das Prinzip Fachhochschule: Erfolg oder Scheitern. Eine Fallstudie am Beispiel Nordrhein-Westfalen. Verlagshaus Monsenstein und Vannerdat OHG Münster.

Hoover-Dempsey, Kathleen V. / Bassler, Otto C. / Brissie, Janet S. (1987): Parent involvement: Contributions of teacher efficacy, school socioeconomic status, and other school characteristics. American Educational Research Journal, 24(3), 417-435. doi:10.3102/00028312024003417.

Hug, Theo / Poscheschnik, Gerald (2015): Empirisch forschen. Die Planung und Umsetzung von Projekten im Studium. 2. Aufl. Konstanz: Verlag Huter \& Roth KG.

Kandemir, Mehmet / Özbay, Yaşar (2012): Akademik Özyeterlik Ölçeği (Aöyö): Geçerlik Güvenirlik Çalışması. In: Erzincan Üniversitesi Eğitim Fakültesi Dergisi, 14(2), 201-214.

Kriegler-Kastelic, Gisela (2018): Selbstwirksamkeitserwartungen. https://infopool. univie.ac.at/ startseite/universitaeres-lehren-lernen/selbstwirksamkeits-erwartungen/ (Letzter Zugriff: 30.06.2021).

Lent, Robert W. u.a. (1986): Self-efficacy in the prediction of academic performance and perceived career options. Journal of Counseling Psychology 33(3), 265-269. doi:10.1037/00220167.33.3.265.

Linnenbrink, Elizabeth A. / Pintrich, Paul R. (2003): The role of self-efficacy beliefs in student engagement and learning in the classroom. Reading \& Writing Quarterly: Overcoming Learning Difficulties, 19(2), 119-137. https://doi.org/10.1080/10573560308223.

Luszczynska, Aleksandra / Gutiérrez-Doña, Benicio/ Schwarzer, Ralf (2005): General self-efficacy in various domains of human functioning: Evidence from five countries. In: Int. J. Psychol., 40, 8089. 10.1080/00207590444000041.

Maddux, James E. / Kleiman, Evan (2021): Self-efficacy. Biswas-Diener, Robert/ Diener, Edward (Hg.): Noba textbook series: Psychology. Champaign, IL: DEF publishers.

Margolis, Howard / McCabe, Patrick P. (2006): Improving self-efficacy and motivation: What to Do, What to Say. In: Intervention in School and Clinic 41(4), 218-227.

McCombs, Barbara / Marzano, Robert (1990): Putting the self in Self-Regulated Learning: The Self as Agent in Integrating Skill and Will. In: Educational Psychologist. 25, 51-70.

Mehmood, Abid u.a. (2019): The effect of self-efficacy on academic performance at higher level of learning: A case study of punjab university lahore. In: Journal of Educational Sciences and Research 6(1), 33-47.

Morales-Rodríguez, Francisco M. / Pérez-Mármol, Jose M. (2019): The role of anxiety, coping strategies, and emotional intelligence on general perceived self-efficacy in university students. Frontiers in psychology 10, 1689. https://doi.org/10.3389/ fpsyg.2019.01689.

Multon, Karen D. / Brown, Steven D. / Lent, Robert W. (1991): Relation of self-efficacy beliefs to academic outcomes: A meta-analytic investigation. Journal of Counseling Psychology, 38(1), 3038. doi:10.1037/0022-0167.38.1.30.

Murphy, P. Karen / Alexander, Patricia A. (2000): A Motivated Exploration of Motivation Terminology. In: Contemporary Educational Psychology 25, 3-53. 
Musa, Matovu (2020): Academic self-efficacy and academic performance among university undergraduate students: An antecedent to academic success. In: European Journal of Education Studies 7(3), 135-149.

Namubiru, Bridget J. (2019): Active learning, student self-efficacy and academic performance among adolescents in secondary schools in kampala district. (Unpublished Master's Dissertation). Makerere University. http://dissertations.mak.ac.ug/handle/ 20.500.12281/7822.

Njega, Stephen W. / Njoka, Johannes N. / Ndung'u, Catherine W. (2019): Assessment of SelfEfficacy on learners' academic performance in secondary schools in Kirinyaga and Murang'a Counties, Kenya. In: Journal of Arts and Humanities, 8(10), 48-59.

Nkechi, Ebizie E. / Ewomaoghene, Enajedu E. / Egenti, Nkechi (2016): The role of guidance and counselling in effective teaching and learning in schools. In: RAY: International Journal of Multidisciplinary Studies, 1(2), 36-48.

Ormrod, Jeanne E. / Anderman, Eric M. / Anderman, Lynley H. (2017): Educational Psychology: Developing Learners (9th Edn.). Harlow-Pearson.

Oyuga, Patricia. A. u.a. (2019): Relationship between self-efficacy and academic performance among orphaned secondary school students in Kenya, International Journal of Psychology and Behavioral Sciences 9(3), 39-46. Doi: 10.5923/j.ijpbs.20190903.02.

Pajares, Frank (1996): Self-Efficacy beliefs in academic settings. Review of Educational Research 66, 543-578.

Petri, Pascale S. (2020): Ein Prozessmodell des Studieneinstiegs: Differentielle Aspekte studiumsbezogener Kognitionen und deren Effekte auf Studienerfolg und Studienabbruch. (Unveröffentliche Disseration). Justus-Liebig-Universität Gießen.

Piergiovanni, Lucia F. / Depaula Pablo D. (2018): Self-Efficacy and stress coping styles in university students. Ciencias Psicológicas 12(1), 17-23. Doi: 10.22235/cp.v12i1.1591.

Sanjuán Suárez, Pilar u.a. (2000): Escala de autoeficacia general: Datos psicométricos de la adaptación para población española [general self-efficacy scale: psychometric data from the adaptation to the Spanish population]. In: Psicothema, 12, 509-513.

Schunk, Dale H. (1990): Goal setting and self-efficacy during self-regulated learning. Educational Psychologist 25, 71-86. doi: 10.1207/s15326985ep2501_6.

Schunk, Dale H. (1991): Self-efficacy and academic motivation. Educational Psychologist 26(3 \& 4), 207-231. Doi: 10.1207/s15326985ep2603\&4_2.

Schunk, Dale H. / Ertmer, Peggy A. (2000): Self-regulation and academic learning: Self-efficacy enhancing interventions. In: Boekartes, Monique u.a. (Hg.): Handbook Self-Regulation. San Diego: Elsevier, 631-649.

Schunk, Dale H. / Pajares, Frank (2009): Self-efficacy theory. In: Wentzel, Kathryn R./ Wigfield, Allan (Hg.): Handbook of motivation at school. New York: Routledge, 35-53.

Schunk, Dale H. / Zimmerman, Barry J. (2006): Competence and Control Beliefs: Distinguishing The Means and Ends. In: Alexander, Patricia A./ Winne, Philip H. (Hg.): Handbook of Educational Psychology. Lawrence Erlbaum Associates, Mahwah, New Jersey, 349-367.

Schwarzer, Ralf / Jerusalem, Matthias (2002): Das Konzept der Selbstwirksamkeit. In Matthias Jerusalem/ Diether Hopf (Hg.), Selbstwirksamkeit und Motivationsprozesse in Bildungsinstitutionen (pp. 28-53). Zeitschrift für Pädagogik: Beiheft; 44. Weinheim u.a.: Beltz.

Seligman, Martin E. P. (1991): Pessimisten küsst man nicht. München: Droemer Knaur. 
Sin, Natasha (2019): College vs. University College vs. University: What's the Difference? https://eduadvisor.my/articles/college-vs-university-college-vs-university-whats-the-difference/ (Letzter Zugriff 15.07.2021).

Stajkovic, Alex / Luthans, Fred (1998): Self-efficacy and work-related performance: A meta-analysis. Psychological Bulletin, 124(2), 240-261. doi:10.1037/0033-2909.124.2.240.

Study in Turkey (2021): Türkiye'de Yükseköğretim Sistemi. https://www.studyinturkey.gov.tr/StudyinTurkey/ShowDetail?rID=i7OVutpiDyU=\&\&cId=PE4Nr 0mMoY4 (Letzter Zugriff 15.07.2021).

Taipjutorus, Widchaporn / Hansen, Sally / Brown, Mark (2012): Investigating a relationship between learner control and self-efficacy in an online learning environment. In: Journal of Open Flexible and Distance Learning, 16(1), 56-69.

Tukey, John W. (1959): A quick, compact, two-sample test to duckworth's specifications. In: Technometrics. 1(1), 31-48.

Valdivieso, Juan A. / Carbonero, Miguel A. / Martín- Antón, Luis J. (2013): The instructional auto perceived competence: A new teaching evaluation scale for its measure in elementary school. In: Journal of Psycho-didactics, 18(1), 47-80.

Valentine, Jeffrey C. u.a. (2004): The relation between self-beliefs and academic achievement: A metaanalytic review. Educational Psychologist 39(2), 111-133. Doi: 10.1207/s15326985ep3902_3.

Wolf, Benjamin M. u.a. (2018): Self-efficacy vs. action orientation: Comparing and contrasting two determinants of goal setting and goal striving. In: Journal of Research Personality 73, 35-45.

YÖK (2019): Türkiye Yükseköğretim Sistemi. https://www.yok.gov.tr/ Documents/Yayinlar/Yayinlarimiz/2019/Higher_Education_in_Turkey_2019_tr.pdf (Letzter Zugriff 15.07.2021).

Yokoyama, Satoru (2019): Academic self-efficacy and academic performance in online learning: A mini review. Frontiers in Psychology 9, 27-94. Doi: 10.3389/fpsyg.2018.02794.

Zimmerman, Barry J. (2000): Self-efficacy: An essential motive to learn. In: Contemporary Educational Psychology 25(1), 82-9.

Zimmerman, Barry J. / Cleary, Timothy J. (2006): Adolescents' development of personal agency: The role of self-efficacy beliefs and self-regulatory skill. Pajares, Frank/ Urdan, Tim (Hg.): Self-efficay beliefs of adolescents. Greenwich, CT: Information Age Publishing, 45-69.

Zimmerman, Barry. J. / Kitsantas, Anastasia (2005): The Hidden Dimension of Personal Competence: Self-Regulated Learning and Practice. In A. J. Elliot/ C. S. Dweck (Hg.), Handbook of competence and motivation Guilford Publications, 509-526. 